\title{
Caveolin-1 Polymorphism (rs7804372) and Cancer Risk: A Meta-Analysis of 15 Case-Control Studies
}

\author{
Juan Wei, Yuqi Qiu, Sufei Wang, Cunjian Yi* \\ Department of Obstetrics and Gynecology, The First Affiliated Hospital of Yangtze University, Jingzhou, China \\ Email:675154204@qq.com,390780721@qq.com, *cunjiany@163.com
}

How to cite this paper: Wei, J., Qiu, Y.Q., Wang, S.F. and Yi, C.J. (2020) Caveolin-1 Polymorphism (rs7804372) and Cancer Risk: A Meta-Analysis of 15 Case-Control Studies. Yangtze Medicine, 4, 208-217. https://doi.org/10.4236/ym.2020.43020

Received: November 5, 2019

Accepted: June 27, 2020

Published: June 30, 2020

Copyright $\odot 2020$ by author(s) and Scientific Research Publishing Inc. This work is licensed under the Creative Commons Attribution International License (CC BY 4.0).

http://creativecommons.org/licenses/by/4.0/

\begin{abstract}
Purpose: Epidemiological studies have assessed the association between Caveolin-1 polymorphism and cancer risk. However, published data are still inconclusive. To clarify this inconsistency, we conducted a meta-analysis to evaluate the relationship between Caveolin-1 polymorphism (rs7804372) and cancer susceptibility. Methods: We conducted a comprehensive literature search, using PubMed, Embase, Medline, Web of Science, CNKI, and Wanfang database, which included English and Chinese literatures. The latest updated date was January 2018. The following search terms were performed to retrieve the relevant articles: ((CAV1) OR (Caveolin-1) OR (rs7804372)) AND (cancer OR tumor OR carcinoma OR neoplasms OR malignancy) AND (polymorphism OR mutation OR variant OR genotype). Odds ratio (OR) with $95 \%$ confidence interval (CI) was used to estimate the pooled effect. Results: In the overall analysis, this kind of polymorphism showed a significant association with increased risk of cancer: allelic model ( $\mathrm{T} / \mathrm{A} ; \mathrm{OR}=1.33,95 \%$ CI: 1.19 - 1.49; $\mathrm{P}<0.0001$ ), homozygous (TT/AA; OR $=1.73,95 \%$ CI: 1.37 2.18; $\mathrm{P}<0.0001$ ), and heterozygous genetic models (TT/TA; OR $=1.23,95 \%$ CI: $1.14-1.33$; $\mathrm{P}<0.0001)$, the dominant genetic model (TT + TA/AA; OR = 1.58, 95\% CI: 1.28 - 1.96; P < 0.0001), and the recessive genetic model (TT/TA $+\mathrm{AA} ; \mathrm{OR}=1.34,95 \% \mathrm{CI}: 1.20-1.50 ; \mathrm{P}<0.0001)$. In addition, the stratified analysis of the results was carried out by ethnicity, HWE status, and cancer types. The outcome indicated that Caveolin-1 rs7804372 polymorphism was associated with an increased risk of cancer. Conclusion: The present study demonstrated that the allele $\mathrm{T}$ of Caveolin-1 (rs7804372) polymorphism might associate with increased susceptibility to cancer, and might predict worse survival in patients with various types of cancer. However, further well-designed studies are required to evaluate this association.
\end{abstract}




\section{Keywords}

Caveolin-1, Polymorphism, Cancer, Susceptibility

\section{Introduction}

As is well known, cancer is one of the leading causes of death in the world [1], neoplasia refers to the complex situation of the organism in various genetic and environmental factors, in which gene mutation plays an important role in genetic factor [2]. Most mutations cause changes in the body, but minor mutations may cause large malignancies. The genetic variants are capable of enhancing cancer development and could be helpful for the early diagnosis, and help in the design of targeted treatment and prevention strategies.

Caveolin-1 (encoded by the CAV-1 gene) is an oncogenic membrane protein associated with endocytosis, extracellular matrix organisation, cholesterol distribution, cell migration and signaling [3]. Caveolin-1 is considered to play a key role in the cell apoptosis and tumor formation [4] [5] [6], and is even considered a tumor suppressor [7] or suppressor depending on tumour type and stage [8].

For the past decade, Cav-1 has been considered a potential biomarker for cancer prognosis, because its expression is upregulated in numerous types of cancer, including breast cancer [9] [10] [11], gastrointestinal tumor [12] [13] [14] [15], urinary neoplasms [16] [17] [18] [19], and other cancers [20] [21] [22]. Available studies show that significant association was found in CAV-1 rs3807987, or rs7804372 gene polymorphism was associated with cancer risk [9]-[22], a recent meta-analysis by Tang et al. showed that CAV-1 rs3807987 polymorphisms may modify the risk of cancer, especially digestive system cancer [23]. But, results for the association of Caveolin-1 polymorphism (rs7804372) and cancer risk were contradictory and inconclusive. In order to solve the problem of the inconsistent results, our study continuously analyzed Caveolin-1 gene rs7804372 for more detailed information between gene polymorphism and cancer susceptibility.

\section{Materials and Methods}

\subsection{Identification and Eligibility of Relevant Studies}

The following databases were searched: PubMed (2000-April, 2018) and Chinese biomedicine literature database (1978-April, 2018) using the following search terms: (Caveolin-1 or CAV-1) and "polymorphism, Genetic" to identify all relevant articles on the subject. Other potential omitted studies were identified by hand screening. The inclusion criteria of studies were as following: 1) studies that evaluated the association between Caveolin-1 polymorphism (rs7804372) and cancer risk, 2) Study provided sufficient data to calculate the odds ratios (ORs) and 95\% confidence intervals (CIs), 3) Case-control study. 


\subsection{Data Extraction}

Using the above criteria for inclusion and exclusion, two independent researchers extracted the information from the included studies. The information mainly included: first author, year of publication, country, ethnicity, tumor type, design of experiment (population-or hospital-based controls) number of genotyped cases and controls, genotypic methods, the characteristics of the controls and quality control. Disagreements were resolved in consultation with the third reviewer. The study quality was assessed according to the NOS [24].

\subsection{Statistical Methods}

The crude ORs and their corresponding 95\% CIs were used to assess the strength of associations between Caveolin-1 polymorphism (rs7804372) and cancer risk. 5 genetic model were used to calculate ORs, including: allelic model ( $\mathrm{T}$ vs $\mathrm{A}$ ), homozygous (TT vs AA), and heterozygous genetic models (TT vs TA), the dominant genetic model (TT + TA vs AA), and the recessive genetic model (TT vs TA + AA). $P$-test (range, $0 \%-100 \%$ ) and chi-square-based Q test were used for assessment of heterogeneity across all selected studies [25]; If $\mathrm{P}<0.1$ or $P>50 \%$, the outcome indicated significant heterogeneity and the random-effects model was used to calculate the pooled OR [25]; otherwise, the fixed-effects model was applied [25]. Hardy-Weinberg equilibrium (HWE) was tested by comparing the observed and expected genotype frequencies of the controls (Chi-square test). Stratified analysis was also conducted according to the type of cancer, ethnicity and HWE (Hardy-Weinberg equilibrium). Publication bias was estimated using Begg's funnel plot [26]. Meta-analysis was performed using Software STATA version 12.0 (STATA Corporation, College Station, TX, USA). P $<0.05$ was considered statistically significant.

\section{Results}

\subsection{Characteristics of Studies}

A total of 15 studies investigating the polymorphism of Caveolin-1 (rs7804372) met our inclusion criteria, including 5893 cases and 6522 controls. These studies were published from 2010 to 2017. In all 15 studies, there were 13 studies of Chinese, remaining two studies were Japanese and Iranians respectively. The all studies included 3 studies on breast cancer, 2 studies on prostate cancer, 4 studies on urinary system neoplasms, 2 studies on gastric cancer, 1 studies on renal cell carcinoma, 1 studies on nasopharyngeal carcinoma, 1 studies on leukemia, 1 studies on oral cancer, 1 studies on hepatocellular carcinoma, 1 studies on esophageal squamous cell carcinoma, 1 studies on upper genitourinary cancer, 1 studies on colorectal cancer, 1 studies on bladder cancer. The distributions of the genotypes in control groups in 6 studies were not in HWE. The Newcastle-Ottawa Scale (NOS) scores of all included studies ranged from 5 to 8 scores (Stang). The characteristics of each study are summarized in Table 1. 
Table 1. Characteristics of included studies.

\begin{tabular}{|c|c|c|c|c|c|c|c|c|c|c|c|c|c|c|}
\hline \multirow{2}{*}{ Auther } & \multirow{2}{*}{ Year } & \multirow{2}{*}{ Country } & \multirow{2}{*}{$\begin{array}{c}\text { Genotype } \\
\text { method }\end{array}$} & \multirow{2}{*}{ SOC } & \multirow{2}{*}{ Ethnicity } & \multirow{2}{*}{$\begin{array}{l}\text { Cancer } \\
\text { type }\end{array}$} & \multicolumn{3}{|c|}{ Case } & \multicolumn{3}{|c|}{ Control } & \multirow{2}{*}{$\frac{\text { HWE }}{\text { P-value }}$} & \multirow{2}{*}{ NOS } \\
\hline & & & & & & & TT & $\mathrm{AT}$ & AA & TT & $\mathrm{AT}$ & AA & & \\
\hline Wang [9] & 2017 & China & PCR & $\mathrm{HB}$ & Chinese & $\mathrm{BC}$ & 317 & 207 & 36 & 338 & 202 & 42 & 0.13 & 8 \\
\hline Fard [10] & 2018 & Iran & RFLP-PCR & $\mathrm{PB}$ & Iranian & $\mathrm{BC}$ & 96 & 65 & 42 & 106 & 74 & 23 & 0.08 & 6 \\
\hline Chang [17] & 2014 & china & PCR & $\mathrm{HB}$ & Chinese & RCC & 29 & 48 & 15 & 285 & 191 & 104 & $<0.01$ & 5 \\
\hline Bau [20] & 2011 & china & RFLP-PCR & $\mathrm{HB}$ & Chinese & OC & 363 & 193 & 64 & 306 & 206 & 108 & $<0.01$ & 8 \\
\hline Hsu [12] & 2013 & china & RT-PCR & $\mathrm{HB}$ & Chinese & $\mathrm{HCC}$ & 166 & 93 & 39 & 152 & 98 & 48 & $<0.01$ & 5 \\
\hline $\operatorname{lin}[13]$ & 2014 & china & RFLP-PCR & $\mathrm{HB}$ & Chinese & GC & 188 & 135 & 35 & 192 & 133 & 33 & 0.16 & 5 \\
\hline liu [11] & 2011 & China & RFLP-PCR & $\mathrm{HB}$ & Chinese & $\mathrm{BC}$ & 745 & 410 & 77 & 694 & 472 & 111 & 0.02 & 5 \\
\hline Sugie [16] & 2013 & japan & RFLP-PCR & $\mathrm{HB}$ & Japanese & PC & 60 & 63 & 11 & 25 & 42 & 19 & 0.86 & 6 \\
\hline Wang [27] & 2014 & China & RFLP-PCR & $\mathrm{HB}$ & Chinese & ESCC & 259 & 143 & 25 & 221 & 166 & 40 & 0.28 & 5 \\
\hline chang [17] & 2013 & China & RFLP-PCR & $\mathrm{HB}$ & Chinese & UUTC & 140 & 65 & 13 & 303 & 224 & 53 & 0.21 & 5 \\
\hline Yang [14] & 2010 & China & RFLP-PCR & $\mathrm{HB}$ & Chinese & CRC & 216 & 117 & 29 & 179 & 120 & 63 & $<0.01$ & 7 \\
\hline Tsou [21] & 2011 & China & RFLP-PCR & $\mathrm{HB}$ & Chinese & NPC & 109 & 55 & 12 & 86 & 57 & 33 & $<0.01$ & 5 \\
\hline Zhang [15] & 2014 & chian & RFLP-PCR & $\mathrm{HB}$ & Chinese & GC & 239 & 134 & 39 & 210 & 136 & 66 & $<0.01$ & 6 \\
\hline bau [18] & 2011 & chian & RFLP-PCR & $\mathrm{HB}$ & Chinese & bladder cancer & 231 & 122 & 22 & 198 & 142 & 35 & 0.2 & 6 \\
\hline wu [19] & 2011 & chian & RFLP-PCR & $\mathrm{HB}$ & Chinese & PC & 163 & 75 & 12 & 254 & 196 & 50 & 0.18 & 6 \\
\hline Wang [22] & 2013 & chian & RFLP-PCR & $\mathrm{HB}$ & Chinese & leukemia & 167 & 86 & 13 & 140 & 99 & 27 & 0.14 & 6 \\
\hline
\end{tabular}

SOC: Source Of Controls; PB: Population-Based Controls; HB: Hospital-Based Controls; HWE: Hardy-Weinberg Equilibrium; NOS: Newcastle-Ottawa Scale; RFLP-PCR: Polymerase Chain Reaction Restriction Fragment Length Polymorphism; BC: Breast Cancer; RCC: Renal Cell Carcinoma; OC: Oral Cancer; HCC: Hepatocellular carcinoma; GC: Gastric Cancer; PC: Prostate Cancer; ESCC: Esophageal Squamous Cell Carcinoma; UUTC: Upper Urothelial Tract Cancer; CRC: Colorectal Cancer; NPC: Nasopharyngeal Carcinoma.

\subsection{Meta-Analysis Results}

The main meta-analysis results and the heterogeneities are shown in Table 2 and Table 3. As shown in the Table 2, all 5 comparisons revealed the association between Caveolin-1 rs7804372 polymorphism and cancer risk after meta-analysis with fixed- or random-effects models. In the overall analysis, this kind of polymorphism showed a significant association with increased risk of cancer: allelic model (T/A; OR = 1.33, 95\% CI: $1.20-1.49 ; \mathrm{P}<0.0001)$, homozygous (TT/AA; $\mathrm{OR}=1.73,95 \% \mathrm{CI}: 1.37-2.18 ; \mathrm{P}<0.0001)$, and heterozygous genetic models (TT/TA; OR $=1.23,95 \%$ CI: $1.14-1.33$; $\mathrm{P}<0.0001$ ), the dominant genetic model (TT + TA/AA; OR $=1.58,95 \% \mathrm{CI}: 1.28-1.96 ; \mathrm{P}<0.0001)$, and the recessive genetic model (TT/TA + AA; OR = 1.34, 95\% CI: $1.20-1.50$; P < 0.0001). In addition, the stratified analysis of the results was carried out by Ethnicity, HWE status, and Cancer types. The outcome indicated that Caveolin-1 rs7804372 polymorphism was associated with an increased risk of cancer (Table 3).

\subsection{Sensitivity Analysis and Publication Bias}

We checked the inclusion criteria of this meta-analysis by a sensitivity analysis. Pooled estimates for all genetic models were insensitive to the removal of individual studies, and the corresponding pooled ORs were not substantially altered (Figure 1), suggesting that our results were stable and reliable.

Begg's funnel plot and Egger's test were performed to assess publication bias (Figure 2). Then, the Egger's test was used to provide statistical evidence of funnel plot symmetry. The results did not show any evidence of publication bias. 
Table 2. Meta-analysis of association between caveolin-1 rs7804372 polymorphisms and cancer risk.

\begin{tabular}{cccccc}
\hline Genetic models & $\begin{array}{c}\text { P for } \\
\text { heterogeneity }\end{array}$ & $\mathcal{F}(\%)$ & Model & OR (95\% CI) & P-value \\
\hline Caveolin-1 rs7804372 & & & & & \\
\hline T vs A & $<0.0001$ & $72.90 \%$ & Random & $1.33(1.19,1.49)$ & $<0.0001$ \\
TT vs AA & $<0.0001$ & $67.20 \%$ & Random & $1.73(1.37,2.18)$ & $<0.0001$ \\
TT vs AT & 0.29 & $14.60 \%$ & Fix & $1.23(1.14,1.33)$ & $<0.0001$ \\
TT vs AT + AA & 0.007 & $54.10 \%$ & Random & $1.34(1.20,1.50)$ & $<0.0001$ \\
TT + AT vs AA & 0.001 & $63.00 \%$ & Random & $1.58(1.28,1.96)$ & $<0.0001$ \\
\hline
\end{tabular}

Table 3. Summary of the subgroup analysis in this meta-analysis.

\begin{tabular}{|c|c|c|c|c|c|}
\hline Comparison & Category & Category & Studies & OR $(95 \% \mathrm{CI})$ & P-value \\
\hline \multirow{8}{*}{ T vs A } & \multirow{2}{*}{ Ethnicity } & Chinese & 13 & $1.36(1.23,1.50)$ & $<0.0001$ \\
\hline & & other & 2 & $1.15(0.45,2.93)$ & 0.77 \\
\hline & \multirow{2}{*}{ HWE } & $\mathrm{Y}$ & 9 & $1.27(1.05,1.53)$ & 0.01 \\
\hline & & $\mathrm{N}$ & 6 & $1.41(1.26,1.57)$ & $<0.0001$ \\
\hline & \multirow{4}{*}{ Cancer type } & breast cancer & 3 & $0.99(0.74,1.32)$ & 0.93 \\
\hline & & Gastrointestinal tumor & 5 & $1.29(1.09,1.53)$ & 0.003 \\
\hline & & Urinary neoplasms & 4 & $1.56(1.36,1.78)$ & $<0.0001$ \\
\hline & & other cancer & 3 & $1.54(1.35,1.77)$ & $<0.0001$ \\
\hline \multirow{8}{*}{ TT vs AA } & \multirow{2}{*}{ Ethnicity } & Chinese & 13 & $1.79(1.49,2.15)$ & $<0.0001$ \\
\hline & & other & 2 & $1.40(0.17,11.18)$ & 0.75 \\
\hline & \multirow{2}{*}{ HWE } & $\mathrm{Y}$ & 9 & $1.58(1.07,2.34)$ & 0.02 \\
\hline & & $\mathrm{N}$ & 6 & $1.88(1.59,2.22)$ & $<0.0001$ \\
\hline & \multirow{4}{*}{ Cancer type } & breast cancer & 3 & $0.98(0.53,1.82)$ & 0.95 \\
\hline & & Gastrointestinal tumor & 5 & $1.65(1.17,2.32)$ & 0.004 \\
\hline & & Urinary neoplasms & 4 & $2.28(1.64,3.18)$ & $<0.0001$ \\
\hline & & other cancer & 3 & $2.27(1.71,3.02)$ & $<0.0001$ \\
\hline \multirow{8}{*}{$\mathrm{TT}$ vs $\mathrm{AT}+\mathrm{AA}$} & \multirow{2}{*}{ Ethnicity } & Chinese & 13 & $1.24(1.14,1.34)$ & $<0.0001$ \\
\hline & & other & 2 & $1.20(0.84,1.70)$ & 0.317 \\
\hline & \multirow{2}{*}{ HWE } & $\mathrm{Y}$ & 9 & $1.26(1.08,1.48)$ & 0.004 \\
\hline & & $\mathrm{N}$ & 6 & $1.23(1.10,1.37)$ & $<0.0001$ \\
\hline & \multirow{4}{*}{ Cancer type } & breast cancer & 3 & $1.08(0.87,1.33)$ & 0.49 \\
\hline & & Gastrointestinal tumor & 5 & $1.17(1.02,1.35)$ & 0.03 \\
\hline & & Urinary neoplasms & 4 & $1.53(1.28,1.84)$ & $<0.0001$ \\
\hline & & other cancer & 3 & $1.30(1.08,1.57)$ & 0.006 \\
\hline \multirow{2}{*}{ TT vs AT } & \multirow{2}{*}{ Ethnicity } & Chinese & 13 & $1.35(1.22,1.50)$ & $<0.0001$ \\
\hline & & other & 2 & $1.24(0.53,2.93)$ & 0.62 \\
\hline
\end{tabular}




\section{Continued}

\begin{tabular}{|c|c|c|c|c|c|}
\hline \multirow{6}{*}{ TT vs AT } & \multirow{2}{*}{ HWE } & $\mathrm{Y}$ & 9 & $1.31(1.08,1.60)$ & 0.006 \\
\hline & & $\mathrm{N}$ & 6 & $1.32(1.23,1.42)$ & $<0.0001$ \\
\hline & \multirow{4}{*}{ Cancer type } & breast cancer & 3 & $1.03(0.79,1.36)$ & 0.81 \\
\hline & & Gastrointestinal tumor & 5 & $1.28(1.13,1.46)$ & $<0.0001$ \\
\hline & & Urinary neoplasms & 4 & $1.64(1.39,1.95)$ & $<0.0001$ \\
\hline & & other cancer & 3 & $1.51(1.27,1.79)$ & $<0.0001$ \\
\hline \multirow{8}{*}{$\mathrm{TT}+\mathrm{AT}$ vs AA } & \multirow{2}{*}{ Ethnicity } & Chinese & 13 & $1.63(1.44,1.86)$ & $<0.0001$ \\
\hline & & other & 2 & $1.22(0.20,7.59)$ & 0.83 \\
\hline & \multirow{2}{*}{ HWE } & $\mathrm{Y}$ & 9 & $1.43(1.01,2.01)$ & 0.04 \\
\hline & & $\mathrm{N}$ & 6 & $1.74(1.48,2.05)$ & $<0.0001$ \\
\hline & \multirow{4}{*}{ Cancer type } & breast cancer & 3 & $0.96(0.53,1.72)$ & 0.89 \\
\hline & & Gastrointestinal tumor & 5 & $1.56(1.14,2.12)$ & 0.005 \\
\hline & & Urinary neoplasms & 4 & $1.57(1.27,1.94)$ & $<0.0001$ \\
\hline & & other cancer & 3 & $1.95(1.41,2.68)$ & $<0.0001$ \\
\hline
\end{tabular}
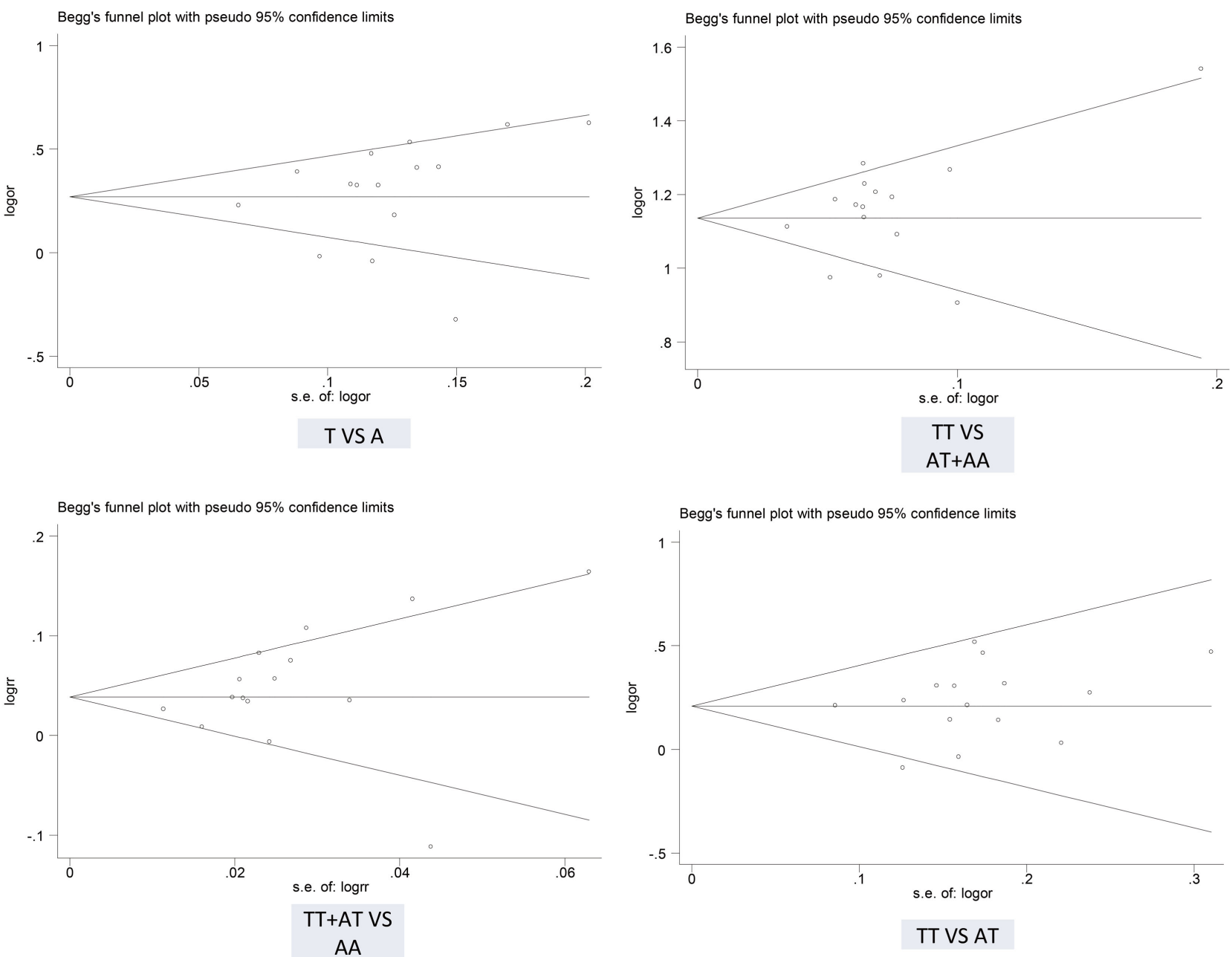

Figure 1. Sensitivity analysis results of the association between caveolin-1 rs7804372 polymorphism and overall cancer risk. Abbreviations: $\mathrm{CI}$, confidence interval; OR, odds ratio. 

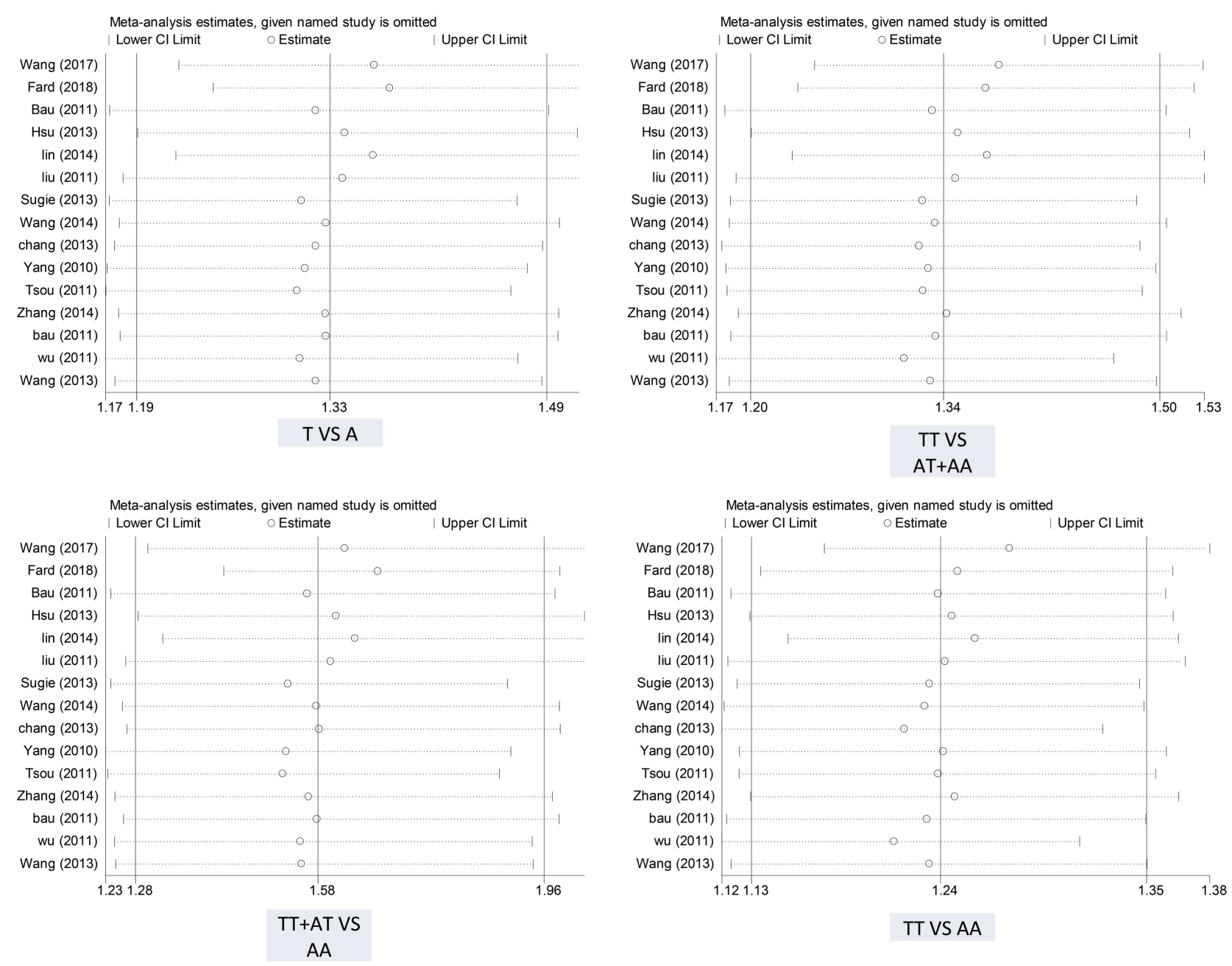

Figure 2. Begg's funnel plots of the association between Caveolin-1 rs7804372 polymorphism and Overall cancer risk. Abbreviations: SE, standard error of the $\log \mathrm{OR}$; $\log \mathrm{OR}$, natural logarithm of the OR; OR, odds ratio.

\section{Discussion}

Located on human chromosome 7 (7q31.1) and contains 3 exons, Caveolin-1 gene can encode the protein of Caveolin-1. CAV-1 is the major structural protein in Caveolin and consists of 178 amino acids, which plays an important role in many signaling pathways, molecular transport, and cellular proliferation and differentiation, potentially involved in the development and metastasis of tumors. In order to figure out controversial results from previous reports, we collected all available published studies and performed a meta-analysis to confirm the association between caveolin-1 (rs7804372) and cancer.

Based on a 15 case-control study, the meta-analysis focuses on the relationship between Asian polymorphism of Caveolin-1 (rs7804372) polymorphism and tumor correlation, and is analyzed by 5 kinds of comparison models. Our study demonstrated caveolin-1 (rs7804372) polymorphism increased the risk of cancer in all five comparison models.

Our meta-analysis had some advantages. For instance, we strictly obeyed the 
inclusion and exclusion criteria to reduce selection bias. We confirmed stratification analysis and sensitivity analysis to seek the sources of high heterogeneity. Stratification analysis was conducted according to ethnicity, HWE status, and Cancer type. Our data indicated Caveolin-1 rs7804372 polymorphism was significantly associated with an increased risk of cancer among Chinese studies. Meantime, statistically significantly increased risks were found among HWE status, and cancer type studies also. However, there were not significantly increased risks of breast cancer in all genetic models. Owing to induce cellular transformation, activate MAPK signaling pathway and alter act in networks, Caveolin-1 rs7804372 polymorphism associates with a higher ER, Her-2 positive rate and tumors size among BC patients (Wangmeng 2017).

Despite the fact that we strictly obeyed the inclusion and exclusion criteria, this meta-analysis may have some limitations. First, publication bias may be present, base of only including data of published studies. Second, All included literatures were case-control studies and have not enough data, we did not perform stratification analysis to clear heterogeneity. Third, some studies do not meet the Hardy-Weinberg equilibrium and cannot fully represent the frequency of genes in the local population. Finally, This meta-analysis mainly based on the Asian population. Therefore, the results can be promoted and applicable to other groups remains unclear.

\section{Conclusion}

The present study demonstrated that the allele T of Caveolin-1 (rs7804372) polymorphism might associate with increased susceptibility to cancer, and might predict worse survival in patients with various types of cancer. However, further well-designed studies are required to evaluate this association.

\section{Conflicts of Interest}

The authors declare no conflicts of interest regarding the publication of this paper.

\section{References}

[1] Torre, L.A., Bray, F., Siegel, R.L., Ferlay, J., Lortettieulent, J. and Jemal, A. (2015) Global Cancer Statistics, 2012. Ca A Cancer Journal for Clinicians, 65, 87-108. https://doi.org/10.3322/caac.21262

[2] Bernstein, C., Anil, R., Nfonsam, V. and Bernstei, H. (2013) DNA Damage, DNA Repair and Cancer. In: New Research Directions in DNA Repair, IntechOpen, London, 413-422. https://doi.org/10.5772/53919

[3] Fra, A.M., Mastroianni, N., Mancini, M., Pasqualetto, E. and Sitia, R. (1999) Human Caveolin-1 and Caveolin-2 Are Closely Linked Genes Colocalized with WI-5336 in a Region of 7q31 Frequently Deleted in Tumors. Genomics, 56, 355-356. https://doi.org/10.1006/geno.1998.5723

[4] Zou, H., Volonte, D. and Galbiati, F. (2012) Interaction of Caveolin-1 with Ku70 Inhibits Bax-Mediated Apoptosis. PLoS ONE, 7, e39379.

https://doi.org/10.1371/journal.pone.0039379 
[5] Lloyd, P.G. and Hardin, C.D. (2011) Caveolae in Cancer: Two Sides of the Same Coin? Focus on "Hydrogen Peroxide Inhibits Non-Small Cell Lung Cancer Cell Anoikis through the Inhibition of Caveolin-1 Degradation". American Journal of Physiology-Cell Physiology, 300, C232-C234. https://doi.org/10.1152/ajpcell.00483.2010

[6] Juhász, M., Chen, J., Tulassay, Z., Malfertheiner, P. and Ebert, M.P. (2003) Expression of Caveolin-1 in Gastrointestinal and Extraintestinal Cancers. Journal of Cancer Research and Clinical Oncology, 129, 493-497. https://doi.org/10.1007/s00432-003-0468-0

[7] Galbiati, F., Volont, D., Engelman, J.A., et al. (2014) Targeted Downregulation of Caveolin-1 Is Sufficient to Drive Cell Transformation and Hyperactivate the p42/44 MAP Kinase Cascade. EMBO Journal, 17, 6633-6648.

https://doi.org/10.1093/emboj/17.22.6633

[8] Gupta, R., Toufaily, C. and Annabi, B. (2014) Caveolin and Cavin Family Members: Dual Roles in Cancer. Biochimie, 107, 188-202. https://doi.org/10.1016/j.biochi.2014.09.010

[9] Wang, M., Tian, T., Ma, X., et al. (2017) Genetic Polymorphisms in Caveolin-1 Associate with Breast Cancer Risk in Chinese Han Population. Oncotarget, 8, 91654-91661. https://doi.org/10.18632/oncotarget.21560

[10] Fard, Z.T. and Nafisi, N. (2018) The Relationship between 6 Polymorphisms of Caveolin-1 Gene and the Risk of Breast Cancer. Clinical Breast Cancer, 18, e893-e898. https://doi.org/10.1016/j.clbc.2018.01.005

[11] Liu, L.C., Su, C.H., Wang, H.C., et al. (2011) Significant Association of Caveolin-1 (CAV1) Genotypes with Breast Cancer in Taiwan. Chinese Journal of Physiology, 54, 153-160. https://doi.org/10.4077/CJP.2011.AMM009

[12] Hsu, C.M., Yang, M.D., Tsai, C.W., et al. (2013) The Contribution of Caveolin-1 Genotype and Phenotype to Hepatocellular Carcinoma. Anticancer Research, 33, 671.

[13] Lin, C.H., Lin, C.C., Tsai, C.W., Chang, W.S., Yang, C.W. and Bau, D.T. (2014) Association of Caveolin-1 Genotypes with Gastric Cancer in Taiwan. Anticancer Research, 34, 2263-2267.

[14] Yang, M.D., Tsai, R.Y., Liu, C.S., et al. (2010) Association of Caveolin-1 Polymorphisms with Colorectal Cancer Susceptibility in Taiwan. World Journal of Gastrointestinal Oncology, 2, 326-331. https://doi.org/10.4251/wjgo.v2.i8.326

[15] Zhang, Y., Hu, X.J., Zhang, L.L., et al. (2014) Interaction among Caveolin-1 Genotypes (rs3807987/rs7804372), H. pylori Infection, and Risk of Gastric Cancer in a Chinese Population. Tumourbiology, 35, 1511-1516. https://doi.org/10.1007/s13277-013-1208-y

[16] Sugie, S., Tsukino, H., Yamauchi, T., et al. (2013) Functional Polymorphism in the CAV1 T29107A Gene and Its Association with Prostate Cancer Risk among Japanese Men. Anticancer Research, 33, 1023-1027.

[17] Chang, W.S., Tsai, C.W., Wang, S.M., et al. (2014) Association of Caveolin-1 Genotypes with Renal Cell Carcinoma Risk in Taiwan. Chinese Journal of Physiology, 57, 220-226. https://doi.org/10.4077/CJP.2014.BAC213

[18] Bau, D.-T. (2011) Significant Association of Caveolin-1 Genotypes with Bladder Cancer Susceptibility in Taiwan. The Chinese Journal of Physiology, 54, 153-160. https://doi.org/10.4077/CJP.2011.AMM009

[19] Wu (2011) Significant Association of Caveolin-1 (CAV1) Genotypes with Prostate Cancer Susceptibility in Taiwan. Anticancer Research, 31, 745-749. 
[20] Bau, D.T., Tsai, M.H., Tsou, Y.A., et al. (2011) The Association of Caveolin-1 Genotypes with Oral Cancer Susceptibility in Taiwan. Annals of Surgical Oncology, 18, 1431-1438. https://doi.org/10.1245/s10434-010-1483-4

[21] Tsou, Y.A., Tsai, C.W., Tsai, M.H., et al. (2011) Association of Caveolin-1 Genotypes with Nasopharyngeal Carcinoma Susceptibility in Taiwan. Anticancer Research, 31, 3629-3632.

[22] O’Donnell, K.A., Yu, D., Zeller, K.I., et al. (2006) Activation of Transferring Receptor 1 by c-Myc Enhances Cellular Proliferation and Tumorigenesis. Molecular \& Cellular Biology, 26, 2373-2386. https://doi.org/10.1128/MCB.26.6.2373-2386.2006

[23] Tang, J., Gang, W., Qiang, L. and Song, R. (2016) Quantitative Assessment of Caveolin-1 G14713A Polymorphism and Cancer Susceptibility in the Asian Population. Oncotargets \& Therapy, 9, 1381-1387. https://doi.org/10.2147/OTT.S92736

[24] Stang, A. (2010) Critical Evaluation of the Newcastle-Ottawa Scale for the Assessment of the Quality of Nonrandomized Studies in Meta-Analyses. European Journal of Epidemiology, 25, 603-605. https://doi.org/10.1007/s10654-010-9491-Z

[25] Higgins, J.P.T. and Thompson, S.G. (2002) Quantifying Heterogeneity in a Meta-Analysis. Statistics in Medicine, 21, 1539-1558. https://doi.org/10.1002/sim.1186

[26] Begg, C.B. and Mazumdar, M. (1994) Operating Characteristics of a Rank Correlation Test for Publication Bias. Biometrics, 50, 1088-1101. https://doi.org/10.2307/2533446

[27] Wang, S., Zhang, C., Liu, Y., Xu, C. and Chen, Z. (2014) Functional Polymorphisms of Caveolin-1 Variants as Potential Biomarkers of Esophageal Squamous Cell Carcinoma. Biomarkers, 19, 652-659. https://doi.org/10.3109/1354750X.2014.968210 